\title{
L'esprit « national » des villes communales des États Pontificaux à la fin du Moyen Âge. Le cas de Bologne
}

\section{Sara Cucini}

\section{OpenEdition}

\section{Journals}

Édition électronique

URL : http://journals.openedition.org/ifha/8034

DOI : $10.4000 /$ ifha.8034

ISSN : 2198-8943

\section{Éditeur}

IFRA - Institut franco-allemand (sciences historiques et sociales)

\section{Édition imprimée}

Date de publication : 31 décembre 2014

ISSN : 2190-0078

\section{Référence électronique}

Sara Cucini, « L'esprit «national » des villes communales des États Pontificaux à la fin du Moyen Âge. Le cas de Bologne ", Revue de l'IFHA [En ligne], 6 | 2014, mis en ligne le 31 décembre 2014, consulté le 21 avril 2019. URL : http://journals.openedition.org/ifha/8034 ; DOI : 10.4000/ifha.8034

Ce document a été généré automatiquement le 21 avril 2019

(CIFHA 


\title{
L'esprit « national » des villes communales des États Pontificaux à la fin du Moyen Âge. Le cas de Bologne
}

\author{
Sara Cucini
}

1 L'émergence d'une culture civique collective, d'une identité culturelle unitaire qui nait autour de la configuration politique urbaine, a été plusieurs fois mise en évidence dans le contexte des villes communales italiennes ${ }^{1}$. À cette émergence, l'historiographie a souvent associé un processus de formation culturelle qui se rapproche de celui de la création d'un sentiment identitaire national : la coniuratio, moment créateur du principe associatif, qui a été définie comme le «mythe fondateur» des communes. La ville communale devient ainsi une collectivité organisée sur une base associative autour d'un principe politique qui, au cours du temps, construit une identité spécifique, fondée sur un passé et une sensibilité religieuse communs et ancrée dans un espace géographique déterminé.

Il existe donc, à tout le moins, une analogie entre la création d'une idéologie civique et la création d'un sentiment national né du " processus de prise de conscience par un peuple du fait qu'il forme une communauté homogène ${ }^{2}$ ». L'association entre la communauté et un principe politique, l'association entre cette même communauté et un territoire défini, le développement d'une historiographie urbaine ${ }^{3}$, l'unité religieuse exprimée par l'émergence de cultes civiques ${ }^{4}$, l'ample utilisation de la rhétorique de la patria et la formation d'une conscience d'appartenance à la communauté ${ }^{5}$, dans certains cas même la revendication d'une identité linguistique spécifique ${ }^{6}$, concourent à la création d'une conscience identitaire des communautés urbaines. Ainsi, la collectivité qui anime la ville ne constitue-t-elle pas une "nation», mais plus certainement une forme d'«identité collective ", dans la création de laquelle nous pouvons retrouver l'application des mêmes outils de construction culturelle qui sont mis en place dans la formation du sentiment «national». La communauté urbaine est fondée sur l'association des citoyens pour 
l'autoreprésentation, et les formes prises par cette autoreprésentation doivent être acceptées et soutenues par cette communauté pour en garantir l'existence. Les dynamiques de construction identitaires sont ainsi utilisées pour la création d'une association indissoluble entre la collectivité et la structure politique qui la représente et doivent être de plus en plus renforcées quand cette structure politique autonome entre en rapport dialectique ou en conflit avec un pouvoir supérieur ou universel. Un exemple de ces dynamiques peut être retrouvé dans l'évolution de l'identité collective de la ville de Bologne. La première attestation de l'évidence d'un sentiment civique dans cette ville est formulée par un texte hagiographique, la vie de saint Pétrone, rédigé au troisième quart du XIIe siècle. Son élaboration se place dans une période pendant laquelle l'autonomie communale était directement mise en danger par l'affrontement avec l'Empire de Frédéric Barberousse. La vie du saint constitue une tentative de susciter la cohésion interne par le lancement d'un nouveau culte ${ }^{7}$. Une autre étape de la construction du sentiment civique bolonais se situe dans une deuxième phase du conflit opposant l'Empire aux autonomies urbaines dans le nord de l'Italie. En 1249, à la suite de la défaite de Fossalta, les Bolonais emprisonnent Enzo, le fils de Frédéric II de Souabe. La ville transfigure en véritable mythe cet épisode, à tel point que l'on a pu parler de " légende hagiographique laïque ${ }^{8}$ ", dont les protagonistes sont la commune et le peuple bolonais. Ainsi, Bologne se dote d'un modèle religieux et d'un mythe agrégateur d'inspiration laïque qui contribuent à la formation d'une identité civique caractéristique. Ces deux passages montrent un processus de formation culturelle mis à jour et réadapté en fonction des variations de la situation politique interne, dans la tentative de rassembler la communauté urbaine autour de sa commune à travers une " orientation " du sentiment collectif.

3 Ne pouvant pas, dans ce contexte, retracer en détail tous les passages évolutifs du sentiment identitaire bolonais, nous nous concentrerons sur une période particulièrement significative. Dès le milieu du XVe siècle, une régularisation des termes de la domination pontificale sur la ville, à laquelle Bologne était formellement soumise depuis $1278^{9}$, s'associe à l'affirmation d'un régime seigneurial sous l'égide de la famille Bentivoglio. Ce régime opère une évolution politique et culturelle supérieure à celle atteinte par les seigneuries personnelles dont la ville avait précédemment fait l'expérience : grâce à ces caractéristiques, l'acceptation et l'intégration de la seigneurie dans le sentiment civique bolonais s'expriment à travers la tentative de constitution d'un modèle dynastique ${ }^{10}$.

4 Une première étape du processus de renouvellement de l'identité communautaire bolonaise est concrétisée par la diffusion de la légende, alimentée par l'entourage bentivolesque, selon laquelle la famille descendrait du roi $\mathrm{Enzo}^{11}$. La parenté présumée avec le fils de Frédéric II permettait aux Bentivoglio de vanter des ancêtres impériaux, mais leur permettait aussi d'instaurer un lien direct avec le protagoniste de la « légende hagiographique laïque » promue comme l'un des deux axes de la création de l'identité collective en pleine période communale. De la même manière, les événements qui conduisent à la prise de pouvoir définitive de la famille, avec Annibale en 1443, se prêtent particulièrement à une interprétation idéologique. La ville se trouvait alors sous la domination de Niccolò Piccinino. Ce dernier, condottiere et délégué des Visconti de Milan, est appelé à Bologne en 1438, pour tenter de mettre fin à une période de domination directe du pontificat. Pourtant, en 1442, Piccinino se rapproche du siège apostolique qui, soutenant les ambitions personnelles du condottiere, essaie de reprendre 
le contrôle de la ville. Annibale Bentivoglio, autour duquel s'étaient rassemblés les forces politiques et le soutien de la population, est alors emprisonné sous un prétexte fallacieux ${ }^{12}$. La libération de Bentivoglio est obtenue grâce à l'intervention d'un autre membre du milieu oligarchique urbain, Galeazzo Marescotti, qui s'introduit furtivement dans le château de Varano, où le Bentivoglio était prisonnier, en permettant sa fuite en juin 1443. Son retour clandestin à Bologne suscite un soulèvement populaire qui aboutit à la libération de la ville ${ }^{13}$.

5 L'importance capitale de ces événements est soulignée par l'abondance de détails présentée par toutes les chroniques bolonaises de la deuxième moitié du XVe siècle. Ces récits mettent en avant la participation aux événements de la population urbaine dans son intégralité. Selon la chronique de Girolamo Borselli, l'emprisonnement du citoyen éminent touche tous les Bolonais qui agissent dès lors comme un seul corps pour tenter de le libérer et lors des conflits qui suivent la rentrée en ville d'Annibale ${ }^{14}$. Le soutien de la population à la cause bentivolesque, présenté comme soutien à la ville et une défense de la liberté bolonaise, est également rappelé par les continuateurs anonymes de la chronique de Bartolomeo della Pugliola. Dans le récit, les continuateurs soulignent par exemple la participation active des femmes au soutien des combattants, en présentant le peuple bolonais comme le protagoniste de toutes les actions militaires contre Niccoló Piccinino ${ }^{15}$.

6 Un autre récit contemporain est présenté par Gaspare Nadi ${ }^{16}$, un membre de l'art des maçons, auteur d'un livre de famille entre 1435 et 1504. Certains passages du texte nous montrent comment, dans la mentalité populaire, le salut et la liberté du peuple bolonais passent par l'action des Bentivoglio. De même, dans le texte de Nadi, la consignation ordinaire des faits relatifs à la vie et à la famille de l'auteur est associée aux récits des événements concernant la famille Bentivoglio. Les dates importantes pour la famille seigneuriale sont désormais aussi considérées comme mémorables par des hommes du milieu populaire, qui s'attachent ainsi de façon significative au sommet de la hiérarchie oligarchique.

7 L'utilisation que la famille fera de l'épisode de 1443, transfiguré en épisode mythique, devient plus évidente aux alentours des années 1480, quand la chronique centrée sur les événements de 1443 et écrite par l'un des protagonistes, Galeazzo Marescotti, est diffusée. Le récit des événements adopte un ton miraculeux : la grâce de Dieu permet au Marescotti et à ses associés de libérer Annibale, la grâce de Dieu garantit au groupe de le reconduire à Bologne sans que personne le reconnaisse. La présence de Bentivoglio à Bologne est présentée comme un catalyseur de l'unité identitaire. La collectivité urbaine s'unit à son seigneur dans la libération de la patria. L'action de Bentivoglio pour la prise du pouvoir est plutôt présentée comme un acte de libération d'une domination extérieure qui avait opprimé jusqu'alors la ville et sa communauté. De même, la lutte de faction ultérieure, qui entrainera l'assassinat d'Annibale Bentivoglio en 1445, dont le récit fait partie intégrante de la chronique, est exposée dans la narration comme la conséquence d'une intervention externe. Dans ce cas aussi, l'auteur souligne la mobilisation non seulement $\mathrm{du}$ parti bentivolesque, mais de toute la population bolonaise, tant de la ville que du contado ${ }^{17}$.

8 La divulgation publique de la chronique survient en $1488^{18}$, dans un contexte de crise du soutien apporté à la seigneurie bentivolesque : la famille Malvezzi, appartenant au cercle oligarchique de la ville, ourdit en 1488 une conjuration, toutefois éventée, contre les Bentivoglio. Cet épisode manifeste ouvertement le développement de l'opposition 
politique contre la seigneurie ${ }^{19}$. Les récits contemporains de la conjuration de 1488 se limitent à une description des faits dépouillée de détails ${ }^{20}$. La rupture de l'unité politique de l'oligarchie urbaine semble se refléter à travers toute la communauté et oblige le Bentivoglio à réunir autour de la famille le consensus de la collectivité urbaine. La reprise $\mathrm{du}$ mythe fondateur du régime bentivolesque, avec le fréquent rappel à l'unité du «peuple bolonais » autour de la famille, permet de réactualiser l'association mise en place au milieu du siècle entre la liberté de la patria et l'action de la branche seigneuriale. La récupération de l'unité patriotique autour du régime bentivolesque était de plus indispensable pour la mise en pratique de la législation en vigueur à Bologne pour châtier les crimes de rébellion. Le régime oligarchique avait profité de l'assassinat d'Annibale Bentivoglio de 1445 pour instituer son organisme de gouvernement comme la seule autorité chargée de désigner les coupables de rebellio ${ }^{21}$. Les seuls cas d'application évidente de ces normes au cours de la période de domination bentivolesque sont à mettre en relation avec la lutte de faction de 1445 et avec la conjuration de $1488^{22}$. Ainsi la loi qui définit les conditions d'exclusion de la communauté expulse ceux qui portent atteinte à la famille seigneuriale.

Bien que la seigneurie ait subi un désaveu pendant les années 1480, elle survivra jusqu'à l'expédition du pape Jules II en 1506. L'objectif de l'action pontificale visait à placer à nouveau Bologne sous le contrôle direct de la souveraineté papale. Ainsi la première action du pape consista-t-elle à décrédibiliser la famille Bentivoglio en maniant l'accusation de crime de lèse-majesté. Au niveau rhétorique, l'action pontificale est présentée comme la libération de la tyrannie établie par les Bentivoglio. L'image que la famille avait essayé de construire, se présentant comme libératrice de la ville et garante de l'indépendance urbaine face à l'oppression externe, est ainsi renversée. Si, pour les Bentivoglio, l'exclusion de la communauté civique est imputable à ceux qui portent atteinte à la famille et à son régime, Jules II bannit de la communauté bolonaise la famille elle-même, au motif qu'elle n'est pas seulement rebelle à la volonté pontificale, mais aussi traitre envers sa propre patrie. Pourtant, l'accusation de lèse-majesté pontificale et la menace de l'excommunication sont adressées non seulement aux membres du gouvernement, mais aussi à tous les citoyens et habitants de Bologne ${ }^{23}$. Ainsi, la " 'croisade' que le pape avait 'publiée' contre Giovanni Bentivoglio, en réalité, visait aussi le peuple et la ville, déclarés rebelles » : en conséquence, la gestion de la crise de 1506 par la ville a été présentée comme une "résistance du peuple » contre les prétentions de domination directe du pontificat et contre la possibilité d'une invasion des troupes françaises qui soutenaient le pontife ${ }^{24}$. Dans ce contexte, le sentiment d'appartenance, dans la sauvegarde de la liberté politique de la ville fondement de la dynamique de constitution identitaire dynastique tentée par les Bentivoglio, ressurgit face aux prétentions pontificales. De cette manière, les instruments de communication politique utilisés par le pontificat pour justifier sa propre action contre la famille seigneuriale sont utilisés par les Bolonais pour justifier leur résistance légitime contre l'action papale. La communauté voit ainsi dans la politique pontificale un élément de déstabilisation, un véritable acte tyrannique visant à restreindre la liberté d'un peuple culturellement uni qui refuse toute influence externe. Le soutien à la famille seigneuriale avait été abandonné à la suite de son éloignement du territoire bolonais au début du mois de novembre 1506. Pourtant, l'identité civique construite de manière «dynastique » par la seigneurie agit au niveau culturel en tant qu'élément d'unité de l'opposition antipontificale. Un exemple de cette attitude peut être cherché dans la diffusion de nombreux textes poétiques d'inspiration polémique ${ }^{25}$, les scrittarini, qui révèlent les sentiments de la 
collectivité civique face aux actions papales. Le scrittarino du Bolonais Ercole Ugolotti, mis à mort pour la diffusion du texte en octobre $1507^{26}$, permet de souligner cet aspect. Selon le scrittarino, la ville, sereine sous la seigneurie bentivolesque, se retrouve soumise aux "prêtres cruels et impitoyables » et menacée par les troupes françaises. Le «peuple bolonais " est donc exhorté à agir pour libérer la ville de la domination considérée comme étrangère, dans l'attente du retour des Bentivoglio attendus le jour de la fête de saint Pétrone. Le texte, incitant la collectivité bolonaise à se rebeller contre la domination pontificale, récupère ainsi l'association entre la famille seigneuriale et la liberté de la ville, en rapprochant ouvertement les Bentivoglio de l'un des éléments constitutifs de l'esprit civique de la période communale.

10 Ainsi, le sentiment d'appartenance civique des villes communales italiennes, qui ne constitue pas un sentiment "national » proprement dit, est-il fondé au cours du temps sur les mêmes outils et le même langage mis en pratique dans la construction de l'idée de «nation ». L'exemple de Bologne montre les circonstances dans lesquelles les outils de construction culturelle sont utilisés, circonstances qui sont dans tous les cas à mettre en relation avec les variations du contexte politique auquel la construction identitaire est associée. Bologne passe donc d'un patriotisme civique fondé sur les aspects religieux et sur la culture juridique communale pendant les XIIe-XIVe siècles à une construction identitaire de type dynastique. Ce passage forme une expérience de construction culturelle qui en tant que telle possède un caractère artificiel, construction avec laquelle on essaye d'identifier l'appartenance à la « patrie » bolonaise avec le soutien et l'adhésion au projet politique de la famille seigneuriale, qui fabrique ce faisant son image d'héritière de la culture politique communale et de garante de la liberté civique. Cette image construite est révélée par la production historiographique urbaine, composant une histoire collective et partagée, mais aussi à travers les expressions plus directes d'un sentiment civique, tel qu'en fournit l'exemple des livres de famille ou celui des scrittarini. Ces écrits démontrent que le sentiment identitaire, y compris dans le contexte des communes italiennes, se construit à travers des «structures rationnelles et émotives, logiques et rhétoriques, incarnées par les actes linguistiques ${ }^{27}$ ».

\section{NOTES}

1. Maria Consiglia De Matteis, "Societas Christiana e funzionalità ideologica della città in Italia : linee di uno sviluppo ", in: Reinhard Elze/Gina Fasoli (dir.), Le città in Italia e Germania nel Medioevo, Bologna : Il Mulino, 1981, p. 13-49.

2. Jean-Marie Moeglin, «Nation et nationalisme du Moyen Âge à l'Époque moderne (FranceAllemagne) », Revue Historique, n 301/3, juillet-septembre 1999, p. 537-553, ici p. 540.

3. Girolamo Arnaldi, «Il notaio-cronista e le cronache cittadine in Italia » in : La storia del Diritto nel quadro delle scienze storiche. Atti del primo Congresso Internazionale della Società Italiana di Storia del Dirtto, Roma 1964, Firenze : Leo S. Olschki, 1966, p. 293-306.

4. Paolo Golinelli, «L'agiografia cittadina : dall'autocoscienza all'auto-rappresentazione (sec. IXXII). Italia settentrionale ", in : Il senso della storia nella cultura medievale italiana, XIV Convegno del 
Centro Italiano di studi di Storia e d'Arte, Pistoia : Centro Italiano di studi di Storia e d'Arte, 1995, p. 253-274.

5. Pietro Costa, Civitas. Storia della cittadinanza in Europa, Roma/Bari : Laterza, 1999, vol. I, p. 21-30 et p. 43-46.

6. Sergio Bertelli, «Egemonia linguistica come egemonismo culturale e politico nella Firenze cosimiana ", Bibliothèque d'Humanisme et Renaissance, n 38/2, mai-août 1976, p. 249-293.

7. Antonio Ivan Pini, « Origine e testimonianze del sentimento civico bolognese », in : La coscienza cittadina nei comuni italiani del Duecento. Atti dell'XI Convegno del Centro Internazione di Studi sulla Spiritualità Medievale, Todi : Accademia Tudertina, 1972, p. 137-193, ici p. 150.

8. Ibid., p. 172.

9. Alfred Hessel, Storia della città di Bologna, in : Gina Fasoli (éd.), Storia della città di Bologna dal 1116 al 1280, Bologna : Alfa, 1975, p. 272-274.

10. Moeglin, « Nation », art. cit., p. 543.

11. Francesca Roversi Monaco, Il comune di Bologna e re Enzo. Costruzione di un mito debole, Bologna : Bononia University Press, 2012, p. 94-96.

12. Angela De Benedictis, «Lo Stato popolare di libertà. Pratica di Governo e Cultura di governo » in: Ovidio Capitani (dir.) Storia di Bologna. 2. Il Medioevo, Bologna : Bononia University Press, 2007, p. 899-950, ici p. 912-913.

13. Cecilia M. Ady, The Bentivoglio of Bologna: a Study in Despotism, London : Oxford University Press, 1937, p. 18-30.

14. Albano Sorbelli (éd.), Cronica gestorum ac factorum memorabilium civitatis Bononie edita a fratre Hyeronimo de Bursellis con la continuazione di Vincenzo Spargiati. Rerum Italicarum Scriptores 2, vol. XXIIIe/2, Città di Castello : Lapi, 1911-1929.

15. Continuatio Chronici Bononiensis per alios scriptores synchrnos, Rerum Italicarum Scriptores, vol. XVIIIe, Milano : Thipographia Societatis Palatina, 1731, colonnes 562-792.

16. Gaspare Nadi, Diario Bolognese, Corrado Ricci/Alberto Bacchi della Lega (éd.), Bologna: Romagnoli dell'Acqua, 1886, p. 14-19.

17. Francesca Roversi Monaco, Conflitti Oligarchici nella Bologna di Annibale I Bentivoglio : la Cronaca di Galeazzo Marescotti de Calvi, Bologna : Clueb, 2012, passim.

18. Cesare Albicini, «Di Galeazzo de' Calvi e della sua cronaca », Archivio Storico Italiano, $\mathrm{n}^{\circ} \mathrm{XXII}$, s. III, 1875, p. 442-460, ici p. 447.

19. Renato Belvederi, «I Bentivoglio e i Malvezzi di Bologna negli anni 1463-1506 », Annali della Facoltà di Magistero. Università degli Studi di Bari, n VI, 1967, p. 33-78, ici p. 63-65.

20. Nadi, Diario Bolognese, op. cit., p. 144-148; Fileno Dalla Tuata, Istoria di Bologna, Bernardo Fortunato (éd.) Bologna : Costa, 2005, vol. I, p. 359-361.

21. Archives d'État de Bologne, Comune-Governo, $n^{\circ}$ 306, 1447 août 9, f. 177-180.

22. Archives d'État de Bologne, Comune-Governo, $n^{\circ} 388$, registre $n^{\circ} 10$, f. 224-226 et ComuneGoverno, $n^{\circ} 401$, registre $n^{\circ} 20$, f. 210-212.

23. Pour le texte de la bulle de condamnation : Luigi Frati, Le due spedizioni militari di Giulio II tratte dal diario di Paride Grassi bolognese, maestro delle cerimonie della cappella papale sui manoscritti di Bologna, Roma e Parigi, con documenti e note, Bologna : Regia Tipografia, 1886, p. 77-186.

24. Angela de Benedictis, Una guerra d'Italia, una resistenza di Popolo. Bologna 1506, Bologna : Il Mulino, 2004, p. 65-66, p. 101-111, p. 123-134, citation p. 111.

25. Giorgio Forni, "La poesia alla guerra di Bologna», in: Gian Mario Anselmi/Angela de Benedictis (dir.), Città in Guerra. Esperienze e Riflessioni nel primo '500. Bologna nelle " Guerre d'Italia ", Bologna : Minerva Edizioni, 2008, p. 229-247, ici p. 229 note. 2.

26. Lodovico Frati, «I Bentivoglio nella poesia contemporanea », Giornale storico della Letteratura Italiana, $\mathrm{n}^{\circ} \mathrm{XLV}, 1905, \mathrm{p} .1-34$, ici p. 30-31.

27. Paolo Evangelisti, «'Misura la città, chi è la comunità, chi è il suggetto, chi è nella città...' Bernardino da Siena, Prediche volgari sul Campo di Siena, XVII, 64 », in : Paolo Prodi/Maria 
Giuseppina Muzzarelli/Stefano Simonetta (dir.), Identità cittadine e comportamenti socio-economici tra Medioevo ed Età Moderna, Bologna : Clueb, 2007, p. 19-52, ici p. 21.

\section{AUTEUR}

\section{SARA CUCINI}

(Université Montpellier 3 Paul-Valéry/Centre d'Études Médiévales de Montpellier EA 4583) 